\title{
Significant new quantitative EEG patterns in fibromyalgia
}

\author{
Jorge Navarro López $z^{a, b, \star}$ \\ Raquel del Moral Bergós ${ }^{a, b}$ \\ Pedro C. Marijuán ${ }^{a, b}$ \\ a Aragon Institute of Health Science (IACS), \\ Zaragoza \\ ${ }^{b}$ Aragon Health Research Institute \\ (IIS Aragón), Zaragoza
}

SPAIN

\begin{abstract}
Background and Objectives: We analyzed the EEG recordings of a sample of fibromyalgia patients, with the goal of looking for new, more objective indicators on the diagnosis and severity assessment of this pathology, and looking also to establish the relationship of these new indicators with different psychological and neuropsychiatric tests.

Methods: We compared the EEG recordings of a group of 13 fibromyalgia patients with a normalized database built into the software of the equipment used (Neuronic), and also with a control group of 13 individuals; both groups were selected under the same criteria of inclusion-exclusion. Patients and controls underwent quantitative EEG (eyes closed), according to international 10-20 EEG system and were specifically evaluated throughout various neuropsychiatric and psychological questionnaires.

Results: We obtained the absolute powers of QEEG (quantitative) for the different electrode sites and frequency bands, we determined the corresponding values of the deviation from normal (Z-scores), and estimated various indicators and ratios, as well as correlations with the results of psychological tests. Interestingly, the ratios of theta and beta frequencies in relation with alpha appear as one of the most relevant indicators of the severity of the pathology; significant differences were also found in the peak frequency (maximum power per $\mathrm{Hz}$ ) of the alpha band, and in the frequency peak of the total spectrum.

Conclusions: The consistency of the abnormal EEG patterns of fibromyalgia patients revealed the presence of systemic dysfunction at the central nervous system level, beyond possible peripheral anomalies and specific tissue pathologies. Among the indicators and benchmarks achieved, the most important changes concern the frequencies theta, alpha and beta, and still more significant were the values of their ratios in the comparison between patients and controls. The relative values of peak frequencies are also of interest. The promising results achieved suggest that it is necessary to continue the investigation to validate these new diagnostic indicators.
\end{abstract}




\section{Introduction}

\section{Description}

Fibromyalgia Syndrome (FM) is a chronic condition of remarkable complexity in its etiology and manifestations. It causes widespread pain and deep exhaustion, with a variety of other symptoms such as restless sleep, paresthesia, stiffness, difficulty in concentration, poor memory, depression, and anxiety ${ }^{1}$. Fibromyalgia mainly affects the soft body tissues and fibrous tissues, such as muscles, tendons, and ligaments. The prevalence of this syndrome reaches about 2-3\% of the population, which means that in a country like Spain the number of affected individuals is in between 800,000 and $1,200,000^{2,3}$. In the same country, it has been found that the prevalence of fibromyalgia in rheumatology clinics is $12 \%$ ( $2.2 \%$ in men and $15.5 \%$ women). Because of its high clinical prevalence, the important impact the disease has on the patient's level of disability, the loss of quality of life, and the associated health expenses it generates, fibromyalgia has become one of the major health problems of Western countries today.

Although the existence of fibromyalgia was accepted some time ago, albeit under different names, it is only recently that the formal criteria for diagnosis have been established. Nowadays diagnosis is developed using the official criteria for fibromyalgia established by the American College of Rheumatology (ACR), where the classification criterion is based on having 11 of the 18 points considered as 'trigger'. These points are recognized as such only if after applying a pressure of $4 \mathrm{~kg} / \mathrm{cm}^{2}$ in a sensitive point, it manifests as painful ${ }^{4}$.

\section{Complex etiology of fibromyalgia}

The significant increase in neurological, physiological and molecular-genetic data about fibromyalgia has not yet resulted in a deeper understanding of its causes. The neurodynamics of this disease, as well as the "psychosomatic" generation of painful processes in general, are far from being elucidated $^{5-7}$. Different methods of imaging (PET, SPECT, functional magnetic resonance, magneto-encephalography) have identified in recent years a complex of structures activated during pain, known as the "pain matrix", in which the thalamus, basal ganglia, insula, anterior cingulate, dorsolateral prefrontal cortex, primary and secondary sensorimotor cortex are involved ${ }^{8,9}$. It has been found that the thalamic activity, which contributes significantly to the processing of pain, is diminished in fibromyalgia. It has also been found that there is a significant decrease in the gray matter of the cortex in fibromyalgia patients, and in the thalamus and prefrontal region of chronic pain patients ${ }^{10,11}$. Further, the glial activation may play a key role in the induction and maintenance of chronic pain ${ }^{12,13}$, as well as in the progressive disruption of the blood brain barrier itself ${ }^{14}$.

In addition to the structural and functional abnormalities in the central nervous system (CNS), we have to add the hypothesis on the hypothalamic-pituitary-adrenal axis dysfunction, the "stress axis", and most likely the involvement of the sympathetic nervous system and the autonomic nervous system. The growth hormone axis would also be abnormally configured in fibromyalgia patients ${ }^{15,16}$. According to M.B. Yunus ${ }^{17}$, this disease could include up to nine different conditions within a common family of "spectrum deregulation syndrome": chronic fatigue, irritable bowel syndrome, stress headaches, migraines, primary dysmenorrheal disorder, periodic limb movement syndrome, restless legs syndrome, temporomandibular pain, and myofascial pain. 


\section{Contributions from EEG to the clinical diagnosis of fibromyalgia}

Given the complexity of the clinical diagnosis of fibromyalgia (FM), which can not always be accurately set, and that the treatment outcome will depend on a dense interplay between physiological, neurological, psychological and social factors, counting with more objective indicators acquires considerable clinical importance. In particular, conspicuous variations in the brain's electrical activity may have an important value as pathological and therapeutic indicators. In the extent to which changes in the CNS are mostly responsible for the development of fibromyalgia $^{18}$, measurements of the neurophysiological activity along the different EEG rhythms may show characteristic abnormalities of this pathological dysfunction.

From the perspective of clinical neurophysiology, and based on quantitative EEG explorations, relevant findings on fibromyalgia have already been reported. The working group on "myosymmetries" in Calgary (Canada) confirmed the existence of subgroups in FM patients for the different brain frequencies $^{19}$. In these patients, the most powerful electrical activity of the brain is placed improperly in the slower waves (slow EEG), with relative deterioration of the alpha rhythm (8-12 Hz) and beta sensorimotor rhythm (12$15 \mathrm{~Hz}$ ). However, that deterioration would not be clear enough but for certain subgroups of patients, depending on different psychological, physiological, and medication factors ${ }^{19}$. On the other hand, more recent works ${ }^{20-22}$, also based on quantitative EEG, have suggested some new elements of discussion in the evaluation of FM patients, from "hypocoherence" in frontal regions to increased beta rhythms in the condition of open eyes, and to increased delta rhythms in sleep.
From the perspective of the present study, the essential aim is to propose some new indicators for diagnosis, in the light of previous studies of EEG too. In our view, rather than the changes in the rhythms themselves, what should be explored is the systematic variation of their ratios-theta vs. alpha and beta vs. alpha being the most relevant. In these two ratios would reside the most significant possibilities for the development of new diagnostic indicators; also in the peak frequencies related with the regionalization of hypocoherence ("cordance") and in the evoked potentials, aspects also addressed in this study, although they will be published separately. The results herein obtained about quantitative EEG ratios look promising enough, and they suggest further research to validate this new class of fibromyalgia indicators on a larger group.

\section{Methodology}

\section{Participants}

We analyzed the EEG records of 26 subjects, in which patients were paired with controls of the same gender and similar ages (in a 13-13 pairing scheme); the same inclusion and exclusion criteria were used for both groups. The sample size was small as corresponding to an exploratory study.

The selected subjects were all women, for gender prevalence in FM is 9:1 female: male. Participant patients suffered fibromyalgia and central fatigue, with a diagnosis of chronic fatigue syndrome (CFS); they were recruited from the Aragon Association of Fibromyalgia and Chronic Fatigue (ASAFA). A total of 26 subjects were selected (13 patients and their 13 age-matched controls), who met the inclusion / exclusion criteria. Inclusion criteria were women aged between 18 and 65, capable of good listening, reading, and compre- 
hension of the instructions, and who met the diagnostic criteria for primary fibromyalgia according to the American College of Rheumatology ${ }^{4}$, and also gave their informed consent to participate in the study. Exclusion criteria were: i) severe psychiatric disorders (dementia, schizophrenia, psychotic disorders, alcohol or substance abuse), anorexia, bulimia nervosa, primary sleep disorders, and obesity; ii) systemic diseases associated with fatigue such as heart failure, chronic respiratory disorders, malignancies, haematological diseases, metabolic diseases, neuromuscular diseases, chronic infections, systemic autoimmune diseases; iii) medical or psychiatric illness or other characteristics that from a clinical point of view discarded the subjects unable to be evaluated psychologically or to carry out the EEG recording session.

A control group matched for sex and age ( \pm 3 years) was selected. This group was composed of women without a diagnosis of chronic fatigue syndrome or fibromyalgia, who were not suffering spontaneous pain and lacked any experience of pain during the month previous to the implementation of the study. Thereafter, 13 subjects aged between 18 and 65 years were included.

During the study, all participants continued with their usual diets and medication unchanged (see Table 1). We followed this ethical considerations supported by recent studies $^{21}$, where it is evident that the different classes of medication used in the treatment of fibromyalgia did not specifically alter the EEG patterns of medicated versus non-medicated patients, beyond the random occurrence (in a large study conducted on 85 patients with fibromyalgia). The multiple medications used in all the different treatments plus the secondary effects due to their combinations were masking the appearance of specific classes, although as a whole group they probably had some effects in the retardation patterns observed.

Table 1

Category of medicaments used by FM patients during EEG recording.

\begin{tabular}{lcc} 
Category & Patients & $\%$ Patients \\
\hline Serotonin Reuptake Inhibitor & 8 & $61.5 \%$ \\
\hline Anti-inflammatory & 7 & $53.8 \%$ \\
\hline Hormonal therapies & 5 & $38.5 \%$ \\
\hline Non-opiate analgesics & 4 & $30.8 \%$ \\
\hline Antispasmoic & 4 & $30.8 \%$ \\
\hline Benzodiazepines & 3 & $23.1 \%$ \\
\hline Osteoporosis & 3 & $23.1 \%$ \\
\hline Tricyclic Antidepressants & 2 & $15.4 \%$ \\
\hline Anti-insomnia & 2 & $15.4 \%$ \\
\hline Opiates & 2 & $15.4 \%$ \\
\hline Gastric motility & 2 & $15.4 \%$ \\
\hline Antiepileptic & 1 & $7.7 \%$ \\
\hline Migraine & 1 & $7.7 \%$ \\
\hline
\end{tabular}




\section{Psychological tests}

Participants were administered various psychological tests and questionnaires to assess psychological constructs related to pain:

- CPAQ: The Chronic Pain Acceptance Questionnaire (CPAQ). It is a questionnaire designed to measure the acceptance of pain ${ }^{23}$.

- CATASTROPHIZATION TO PAIN: This questionnaire evaluates a negative and exaggerated orientation towards pain stimuli. Its Pain Castrophizing Scale (PCS) comprises three subscales: rumination, magnification and helplessness. We used the Spanish validated scale ${ }^{24}$.

- STAI: State Trait Anxiety Inventory (STAI). It is an inventory designed to evaluate two independent concepts of anxiety ${ }^{25}$ : anxiety as state (transient emotional condition) and trait anxiety (anxious relatively stable propensity).

- BDI: The Beck Depression Inventory (BDI). It is a questionnaire that evaluates the clinical symptoms and intrusive thoughts present in depression. This is the scale of depression that presents the highest percentage of cognitive items ${ }^{26}$.

- SF36: This questionnaire is an instrument to assess quality of life related to health, conceptually based on a two-dimensional model of health, physical and mental ${ }^{27}$.

\section{EEG recording}

Informed consent was obtained from all patients and controls before the beginning of the study. Participants had been previously informed of the objectives and characteristics of the study. The research protocol followed the norms approved by the Ethics Committee of the Aragon Institute of Health Sciences (CEICA), Act No. 06/2009.
The quantitative EEG was recorded in both fibromyalgia patients and control subjects under the following conditions: at rest, closed eyes, on high alert, and sitting down comfortably in a soundproof room and dim light. An assemblage with 19 electrodes (International System 10-20) was used, referenced to both earlobes.

The EEG signal was recorded by means of a Neuronic $^{\circledR}$ electroencephalograph. A QEEGT ${ }^{\circledR}$ software (Quantitative EEG Tomographic) version 6.0 of the same brand was used to calculate the absolute and relative power for the different frequency bands. The recorded signal was digitized at $256 \mathrm{~Hz}$, with a filter of $0.3 \mathrm{~Hz}$ for low frequency, and $35 \mathrm{~Hz}$ for high frequency; impedances of all electrodes were maintained at all times below $5 \mathrm{k} \Omega$.

The EEG records were stored separately for each subject, and then analyzed individually on the computer to detect eye movements, muscle artifacts, and possible changes in alertness. The signal from each EEG channel was digitized at 128 samples/s. A trained technician reviewed each record and selected one minute free of artifacts for each subject ${ }^{28}$. These selected periods were spectrally processed using the Fast Fourier Transform method to calculate the absolute power (energy intensity in microvolts squared) for each of the four frequency bands $(0.5-4 \mathrm{~Hz} 4-8 \mathrm{~Hz}$, $8-12 \mathrm{~Hz}$ and $12-20 \mathrm{~Hz}$ ) by means of the already mentioned QEEGT ${ }^{\circledR}$ software (Quantitative EEG Tomographic) version 6.0.

\section{Data analysis}

The QEEG data processed by the Fast Fourier Transform (FFT) provided absolute and relative power scores for the four frequency bands and for each of the electrodes. Additionally, Z scores were obtained for each subject comparing with a normal population. 
These $\mathrm{Z}$ scores express the distance between the patient and control data and the average value of the normal population (standardized between 5 and 90 years) as incorporated in the Neuronic ${ }^{\circledR}$ system. That distance is measured in number of standard deviations; and $Z$ scores greater than 2 or less than -2 standard deviations $(Z= \pm 1.96)$ are sufficient to say that a patient's data are outside from the normal population, with a risk of error of $5 \%$ (probability < 0.05 ). If, conversely, the $\mathrm{Z}$ scores calculated for a patient are in the range $[-1.96,1.96]$ it can be said that the patient belongs to the normal population (in the calculated parameters) with probability of 0.95 (http:// www.icneuronic.com).

Therefore, $\mathrm{Z}$ scores were obtained in all patients and controls for the QEEG absolute and relative powers, as well as for the scores of the peak frequency (maximum output) in the alpha band and in the total spectrum.

\section{Statistic analysis}

A descriptive analysis of the quantitative variables obtained by the EEG was performed for the whole population by determining the mean and standard deviation (SD) as well as the deviation from normality in Z-scores, as discussed above. For comparison between samples, the statistical significance was obtained by the t-Student.

In order to assess the degree of correlation between the quantitative parameters obtained from different EEG and psychological tests, Pearson correlation coefficients were calculated. The analyses were performed using SPSS for Windows, version 15.0.

\section{Results}

\section{Demography}

Demographic results of participants indicate that they all were women $(n=26)$, with an average age for patients of $55.69(\mathrm{SD}=8.00)$ years, and for controls $57.30(\mathrm{SD}=7.83$ ) years. All patients had suffered pain and symptoms of fibromyalgia for at least three years, having been diagnosed with this disease repeatedly.

\section{Psychological outcomes}

The results obtained for the battery of psychological assessment tests are shown in Table 2.

There appeared significant differences between patients and controls concerning levels of depression and also in the perception of health and quality of life. All the 13 patients showed from mild to moderate depression, with an average score equal to 8 , which is the cutoff for the diagnosis of moderate depression, while the average score of controls was 3.5 , which corresponds to absence of depression or minimal depression. Regarding the catastrophizing variable (PCS), that is, the set of cognitive and emotional processes that predispose to the development of chronic pain, no statistically significant differences between patients and controls were found in this study. The differences found in pain acceptance variable (CPAQ) between patients and controls were not statistically significant, probably due to the variability of the results obtained in controls $(\mathrm{SD}=19.07)$ and also to the reduced sample size. Similarly, there were not statistically significant differences in the anxiety variable among patients and controls, even though a regular feature of these patients is to perceive situations as threatening or anxiety-inducing. 
Table 2

Results obtained for the psychological assessment.

\begin{tabular}{lcccc} 
Test & Patients $(\sigma)$ & Controls $(\sigma)$ & Variation $\%$ & p-valor \\
\hline CPAQ activities engagement & $31.92(11.43)$ & $38.30(18.43)$ & -17 & $\mathrm{NS}$ \\
\hline CPAQ pain willingness & $20.85(9.04)$ & $29.00(19.07)$ & -28 & $\mathrm{NS}$ \\
\hline CPAQ total & $52.77(13.55)$ & $67.30(27.81)$ & -22 & $\mathrm{NS}$ \\
\hline STAI state & $36.00(26.32)$ & $18.70(28.87)$ & 93 & $\mathrm{NS}$ \\
\hline STAI trait & $54.83(32.12)$ & $36.50(30.62)$ & 50 & $\mathrm{NS}$ \\
\hline BDI & $8.00(4.56)$ & $3.50(4.04)$ & 129 & $\mathrm{p}<0.05$ \\
\hline Catastrofizing $($ PCS $)$ & $16.17(9.61)$ & $12.10(11.42)$ & 34 & $\mathrm{NS}$ \\
\hline SF36 physical functioning & $46.25(16.67)$ & $86.00(8.76)$ & -46 & $\mathrm{p}<0.05$ \\
\hline SF36 bodily pain & $36.25(15.66)$ & $68.10(15.59)$ & -47 & $\mathrm{p}<0.05$ \\
\hline SF36 general health & $43.33(16.28)$ & $72.50(12.08)$ & -40 & $\mathrm{p}<0.05$ \\
\hline SF36 vitality & $22.50(15.30)$ & $68.00(19.32)$ & -67 & $\mathrm{p}<0.05$ \\
\hline SF36 social functioning & $58.33(27.35)$ & $90.00(16.46)$ & -35 & $\mathrm{p}<0.05$ \\
\hline SF36 mental health & $65.33(23.77)$ & $75.60(22.82)$ & -14 & $\mathrm{p}<0.05$ \\
\hline
\end{tabular}

NS: No significance.

Fibromyalgia patients significantly referred having poorer quality of life and lower self-perceived health than participants without the disease (see Table 2, SF36 items). These differences are observed in all dimensions studied through the questionnaire SF36, especially, in the degree in which they perceive that their poor health limits their daily life activities and work performance, also conditioning the intensity of perceived pain, the lack of vitality and energy, and the sense of discouragement and tiredness.

\section{EEG results}

As an illustration of the results obtained, a comparative of the QEEG scores for a patient and her paired control are shown first (in Figure 1). These data, associated to the absolute powers, exemplify the overall results of declining powers for almost all frequency bands, but in different ways between them. The declining power can be seen in the pictures at the top row, where the absolute powers for each frequency are represented, indicating also the total power on the right side; the green and blue colors stand for powers below the average of the standard reference population, while orange and red colors stand for scores above that average. The bottom row shows the same images than in the top row, but now they are directly referred to their deviation from the normal population as Z-scores of clinical standards included in the analysis software (www.icneuronic.com).

In Figure 1a) the patient $P 1$ shows scores of absolute powers that are clearly below normal standards; this occurs in most brain regions, for both the alpha and theta frequency and for the total power as well. The Z-scores $( \pm 2)$ below indicate the same phenomenon, even more clearly, always referred 


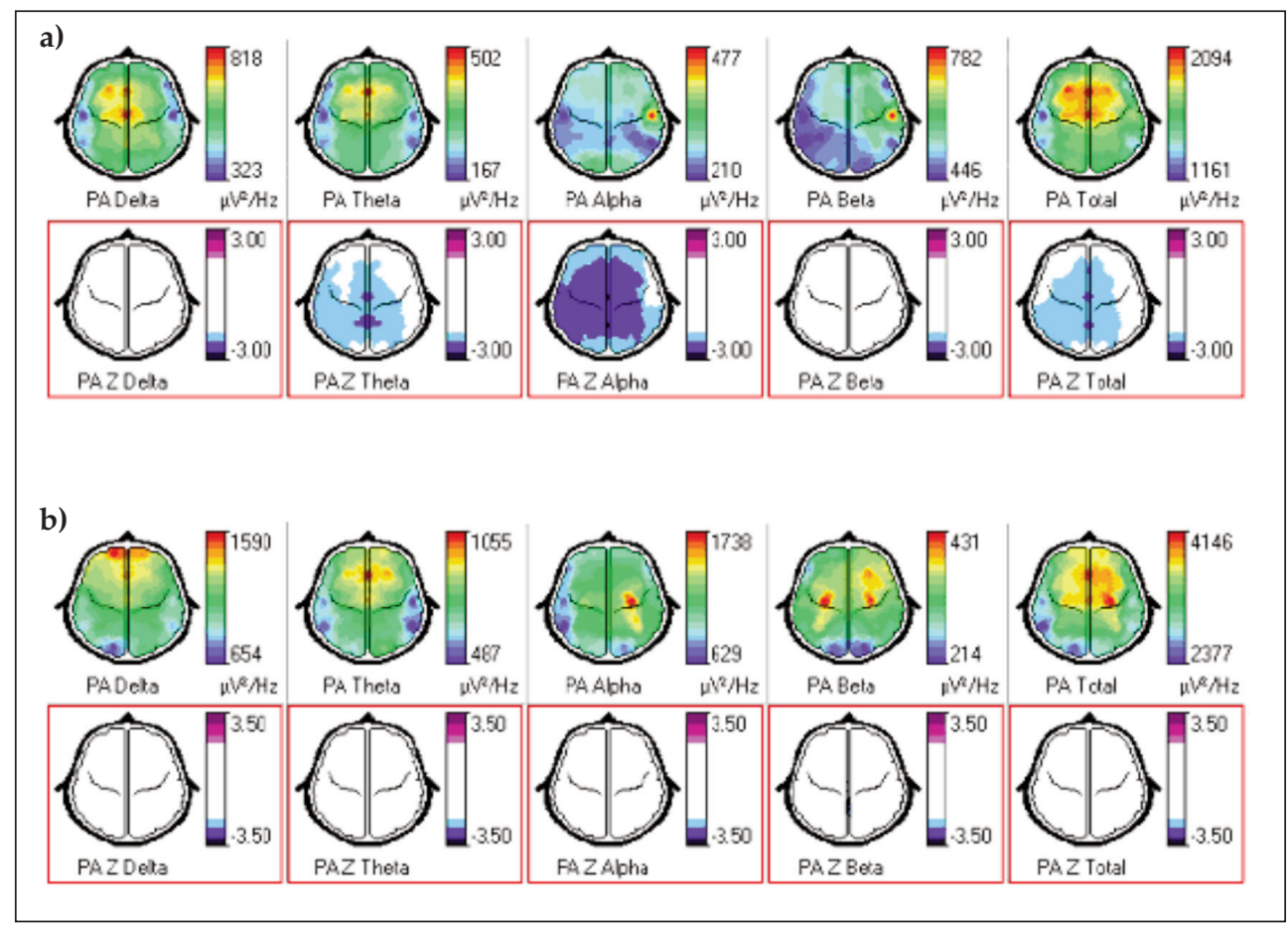

Figure 1. EGG values representing patient $P 1$ and control $C 1$. a) In the top row, $P 1$ values of absolute power for each frequency, with the total power on the right side; the lower row shows for each of the absolute powers deviation from a normal population (Z-scores), according to clinical standards included in the analysis software. b) Similar QEEG parameters representation for the control $C 1$.

to the standards incorporated in the population of the Neuronic ${ }^{\circledR}$ system. It has to be compared with Figure 1b), which contains the same data, but now corresponding to a subject control. In the control $C l$ the recorded power scores do not deviate from the normal (Z-scores \pm 2 ), according to the same standards incorporated in the QEEG software of the Neuronic system.

Figure $2 \mathrm{a}$ ) and b) show similar scores, but now they correspond to averages of the patient group and the control group. They have been obtained by using Matlab EEGlab toolkit on the individual scores. To observe that power in beta frequency is slightly increased in the patient's population respect the controls.
Although the differences between patients and controls with respect to the scores of absolute power for the different frequencies are significant by themselves, it is the change of the ratios between pairs of frequencies what becomes more significant and demonstrative of the QEEG differences between patients and controls.

In Table 3 we represent the results obtained for all patients, in terms of absolute powers, shown as deviations from normal (Z-scores), distinguishing the scores for the different frequency bands and for each of the electrode locations. Also, in the last row, there is a summary of the average power for each of the frequencies and for the total spectrum. 


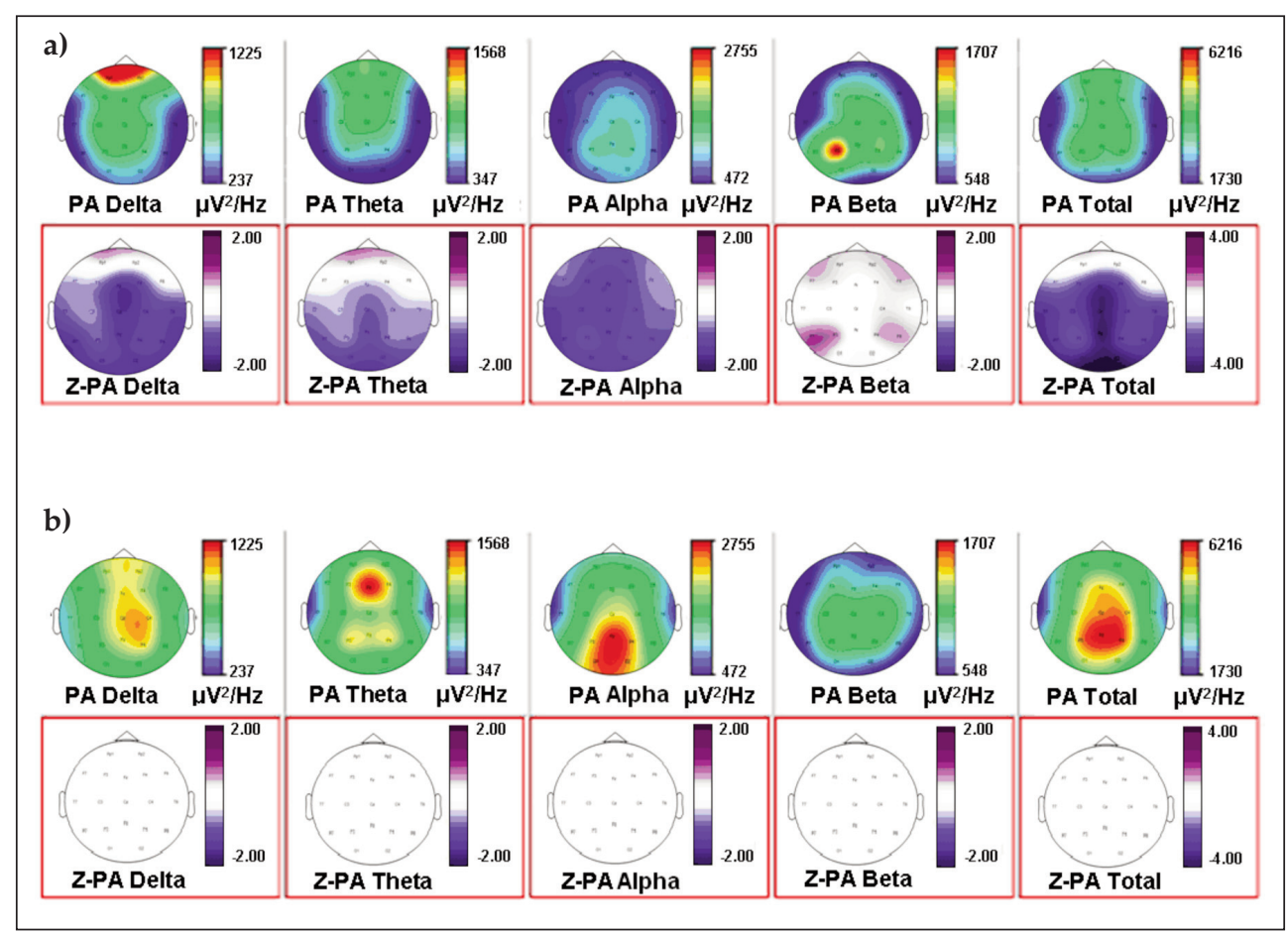

Figure 2. EGG values representing averages of patients a), versus controls b).

To note that power in beta frequency is slightly increased in the patient average respect the controls.

Comparing the scores of the absolute deviations, the highest differences correspond to the alpha frequency, as evidenced by the most negative Z-scores in Table 3 (average alpha -1.02 , versus scores for other frequencies: $-0.92,-0.55,0.23)$. However, for the beta frequency we infer a general increase of absolute power in almost all electrodes, as evidenced by the positive $\mathrm{Z}$-scores for absolute power (beta average 0.23 compared to negative scores of all other frequency bands). That is, the inspection of Table 3 evidences that the power of the alpha band has shifted to the other bands, particularly towards the two neighboring bands beta and theta.

This becomes even more apparent in Figure 3, where the absolute powers of the patient group and the control group of Figure 2 are represented comparatively, by superimposing their respective averages for delta, theta, alpha, and beta frequencies. A general decrease in the absolute power of patients versus controls may be observed, although more pronounced in alpha, and slightly increased in beta.

Table 4 shows the absolute power variations for both patients and controls, corresponding to each frequency band and to the total spectrum. The latter, in the case of patients, represents a value of $3059.32 \mu \mathrm{V}^{2} / \mathrm{Hz}$, whereas controls have $4739.43 \mu \mathrm{V}^{2} / \mathrm{Hz}$, resulting thus in a $35 \%$ decrease in patients $(\mathrm{p}<0.05)$. The biggest change relates to the alpha frequency, with scores of $821.58 \mu \mathrm{V}^{2} / \mathrm{Hz}$ (patients) and $1858.72 \mu \mathrm{V}^{2} / \mathrm{Hz}$ (controls), implying a decrease of $56 \%(\mathrm{p}<0.05)$. The delta and theta 
Table 3

Representation of the patients' absolute power for each of the frequency bands and each of the 19 electrodes, with values of the Z-scores deviation from normal. A summary with the average deviations from normal in each of the frequency bands and the total for the patient group appears on the bottom line.

\begin{tabular}{lccccc} 
CHANNELS & Delta Z & Theta Z & Alpha Z & Beta Z & TOTAL Z \\
\hline Fp1 & 0.26 & 0.39 & -0.95 & 0.32 & 0.02 \\
\hline Fp2 & -0.04 & 0.26 & -0.96 & 0.34 & -0.40 \\
\hline F3 & -0.68 & -0.37 & -1.14 & 0.25 & -1.94 \\
\hline C3 & -1.04 & -0.34 & -1.02 & 0.22 & -2.18 \\
\hline C4 & -0.64 & -0.55 & -1.16 & -0.06 & -2.41 \\
\hline P3 & -1.12 & -0.64 & -0.95 & 0.29 & -2.42 \\
\hline P4 & -0.85 & -0.67 & -0.95 & 0.58 & -1.89 \\
\hline O1 & -1.12 & -0.75 & -0.91 & 0.41 & -2.37 \\
\hline O2 & -1.20 & -1.15 & -1.15 & 0.05 & -3.45 \\
\hline F7 & -1.32 & -1.17 & -1.07 & -0.13 & -3.69 \\
\hline F8 & -0.54 & -0.13 & -0.79 & 0.39 & -1.07 \\
\hline T3 & -0.21 & 0.01 & -0.64 & 0.43 & -0.41 \\
\hline T4 & -0.84 & -0.67 & -1.26 & -0.10 & -2.87 \\
\hline T5 & -1.18 & -0.59 & -0.76 & 0.13 & -2.40 \\
\hline T6 & -1.35 & -0.89 & -1.20 & 0.77 & -2.67 \\
\hline FZ & -1.25 & -0.76 & -0.93 & 0.51 & -2.43 \\
\hline CZ & -1.42 & -0.58 & -1.24 & 0.06 & -3.18 \\
\hline PZ & -1.48 & -0.88 & -1.17 & 0.01 & -3.52 \\
\hline Average & -1.37 & -1.05 & -1.17 & -0.13 & -3.72 \\
\hline
\end{tabular}

Table 4

Values of QEEG absolute powers $\left(\mu \mathrm{V}^{2} / \mathrm{Hz}\right)$ in patients and controls, for each of the frequency bands and for the total spectrum.

\begin{tabular}{lcccc} 
Frequency & $\mathrm{AP}_{\text {patients }}(\sigma)$ & $\mathrm{AP}_{\text {controls }}(\sigma)$ & Variation $(\%)$ & p-value \\
\hline Delta $(0-4 \mathrm{~Hz})$ & $605.41(148.82)$ & $886.58(172.32)$ & -32 & $<0.05$ \\
\hline Thetha $(4-8 \mathrm{~Hz})$ & $685.17(180.05)$ & $1107.05(257.27)$ & -38 & $<0.05$ \\
\hline Alpha $(8-12 \mathrm{~Hz})$ & $821.58(323.52)$ & $1858.72(725.05)$ & -56 & $<0.05$ \\
\hline Beta $(12-20 \mathrm{~Hz})$ & $946.56(117.91)$ & $885.87(123.98)$ & 7 & $<0.05$ \\
\hline Total $(\mathbf{0 - 2 0 H z})$ & $3059.32(729.17)$ & $4739.43(1038.12)$ & -35 & $<0.05$ \\
\hline
\end{tabular}



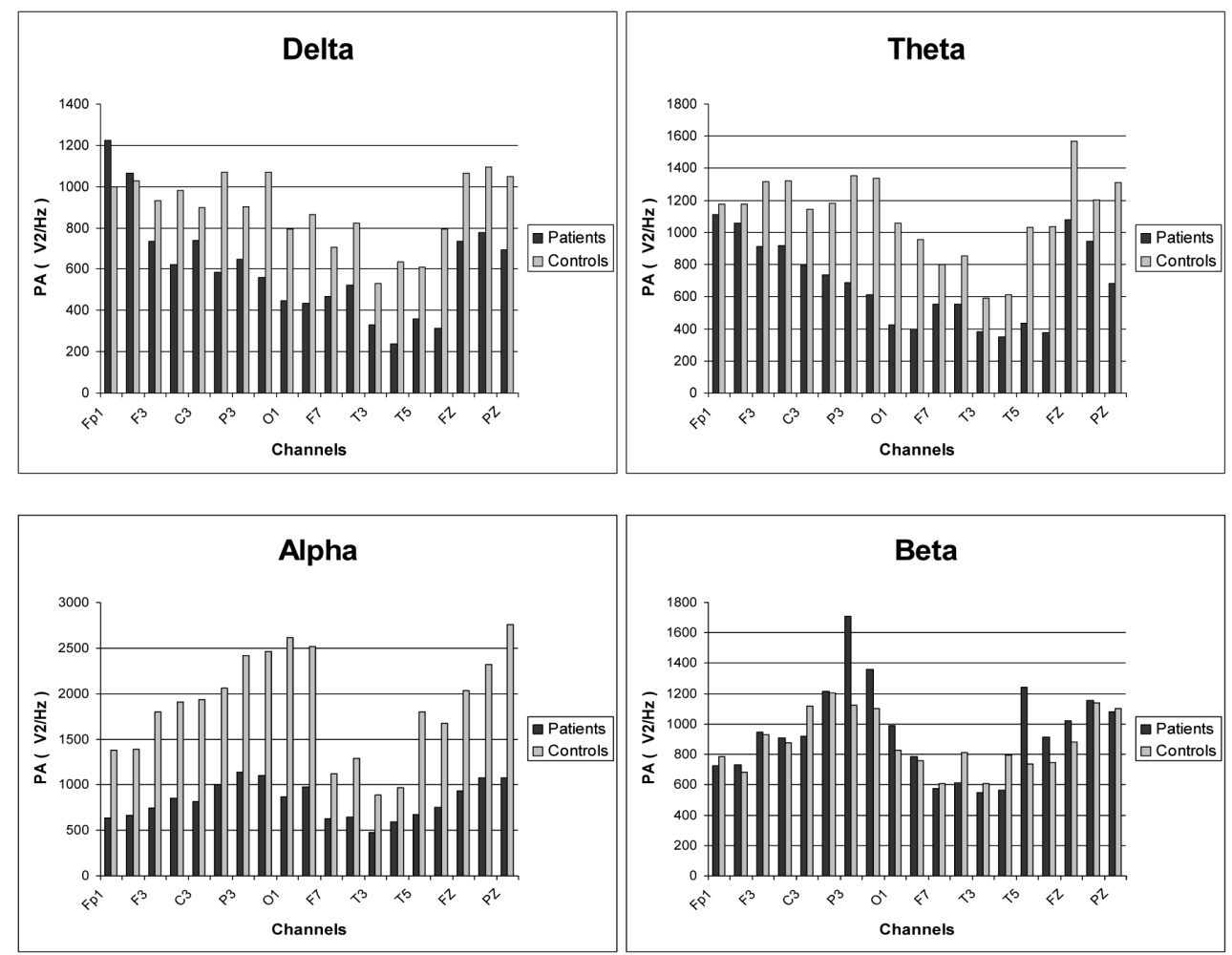

Figure 3. Values of the mean absolute powers of patients (black color) compared to their controls (grey color), for each of the frequencies and electrodes.

frequencies also suffer a decrease of $32 \%$ and $38 \%(\mathrm{p}<0.05)$ respectively. However, for beta frequencies, we find a value of 946.56 $\mu \mathrm{V}^{2} / \mathrm{Hz}$ in the case of patients, while controls have $885.87 \mu \mathrm{V}^{2} / \mathrm{Hz}$, implying an increase of $7 \%$ for patients $(\mathrm{p}<0.05)$.

In view of the contrasting scores obtained, we proceeded to calculate systematically for all patients and controls the average scores of theta vs. alpha and beta vs. alpha ratios in each electrode. These average scores are shown in Table 5. There appear increases of $38 \%$ and $140 \%$ in the respective ratios between total averages (last row of the table). That is, patients have markedly increased their ratios relative to controls, thereby magnifying the effects of displacement activity from alpha to theta and beta. A marked regionalization of the effects occurs in the occipital and temporal areas (especially in the ratio of beta vs alpha); and also in the frontal and prefrontal localizations. The statistical significance of the comparison of these two ratios for patients and controls is highly significant $(\mathrm{p}<0.01)$.

The scores obtained for the peak frequencies in the alpha band and total spectrum show the following distribution:

- Alpha peak frequency: mean patients 9.19 $(\mathrm{SD}=2.03)$, controls $8.81(\mathrm{SD}=0.68)$;

- Whole spectrum peak: mean patients 3.81 $(\mathrm{SD}=4.13)$, controls $2.91(\mathrm{SD}=3.81)$. 
Table 5

Comparison of average values for ratios theta vs. alpha and beta vs. alpha in each of the electrodes, both for patient and control groups $(\mathrm{p}<0.01)$

\begin{tabular}{|c|c|c|c|c|c|c|}
\hline CHANNELS & $\begin{array}{l}\text { Patients } \\
\text { Theta/Alpha }\end{array}$ & $\begin{array}{c}\text { Controls } \\
\text { Theta/Alpha }\end{array}$ & Variation $\%$ & $\begin{array}{c}\text { Patients } \\
\text { Beta/Alpha }\end{array}$ & $\begin{array}{c}\text { Controls } \\
\text { Beta/Alpha }\end{array}$ & Variation $\%$ \\
\hline Fp1 & 1.76 & 0.85 & 107 & 1.14 & 0.57 & 100 \\
\hline Fp2 & 1.59 & 0.85 & 87 & 1.10 & 0.49 & 124 \\
\hline $\mathrm{F} 3$ & 1.22 & 0.73 & 67 & 1.27 & 0.52 & 144 \\
\hline $\mathrm{F} 4$ & 1.07 & 0.69 & 55 & 1.06 & 0.46 & 130 \\
\hline $\mathrm{C} 3$ & 0.97 & 0.59 & 64 & 1.12 & 0.58 & 93 \\
\hline $\mathrm{C} 4$ & 0.74 & 0.57 & 30 & 1.22 & 0.59 & 107 \\
\hline P3 & 0.61 & 0.56 & 9 & 1.50 & 0.46 & 226 \\
\hline P4 & 0.56 & 0.54 & 4 & 1.23 & 0.45 & 173 \\
\hline $\mathrm{O} 1$ & 0.49 & 0.41 & 20 & 1.14 & 0.32 & 256 \\
\hline $\mathrm{O} 2$ & 0.41 & 0.38 & 8 & 0.81 & 0.30 & 170 \\
\hline F7 & 0.89 & 0.71 & 25 & 0.92 & 0.54 & 70 \\
\hline F8 & 0.87 & 0.66 & 32 & 0.95 & 0.63 & 51 \\
\hline T3 & 0.80 & 0.67 & 19 & 1.16 & 0.68 & 71 \\
\hline $\mathrm{T} 4$ & 0.59 & 0.63 & -6 & 0.96 & 0.82 & 17 \\
\hline $\mathrm{T} 5$ & 0.65 & 0.57 & 14 & 1.85 & 0.41 & 351 \\
\hline T6 & 0.50 & 0.62 & -19 & 1.22 & 0.45 & 171 \\
\hline $\mathrm{FZ}$ & 1.16 & 0.77 & 51 & 1.10 & 0.43 & 156 \\
\hline $\mathrm{CZ}$ & 0.88 & 0.52 & 69 & 1.07 & 0.49 & 118 \\
\hline PZ & 0.64 & 0.48 & 33 & 1.01 & 0.40 & 153 \\
\hline Totales & 0.83 & 0.60 & 38 & 1.15 & 0.48 & 140 \\
\hline
\end{tabular}

Finally, about the correlations between the QEEG results and the psychological tests, Table 6 shows the Pearson correlations found statistically significant corresponding to the 13 patients. To emphasize the correlations found between catastrophizing (PCS) and the Z-scores for absolute powers in the beta band $(0.627 ; \mathrm{p}=0.029)$ and the total spectrum
$(0.607 ; \mathrm{p}=0.036)$. The ratio theta vs. alpha correlated significantly with Z-delta PA (-0.753; $\mathrm{p}=0.003$ ), apart obviously from its correlation with Z-alpha and Z-theta. The ratio beta vs. alpha correlates with Z-delta $(-0.597 ; \mathrm{p}=$ $0.031)$ and Z-theta $(-0.612 ; p=0.026)$. See Table 6 for all the correlations found in QEEG indicators. 
Table 6

Pearson correlations between the indicators obtained from the quantitative EEG and the PCS test (catastrophizing).

\begin{tabular}{llccccc} 
& & Theta/Alpha & Beta/Alpha & PCS & Peak $_{\max }(\mathrm{Hz})$ & Peak $_{\text {alpha }}(\mathrm{Hz})$ \\
\hline Z-Delta & Pearson & $-0.753^{* *}$ & $-0.597^{*}$ & -0.433 & 0,139 & 0,235 \\
& Signi. (p) & 0.003 & 0.031 & 0.160 & 0,651 & 0,440 \\
\hline Z-Theta & Pearson & $-0.712^{* *}$ & $-0.612^{*}$ & 0.386 & 0,248 & 0,010 \\
& Signi. (p) & 0.006 & 0.026 & 0.325 & 0,414 & 0,974 \\
\hline Z-Alpha & Pearson & $0.823^{* *}$ & $0.685^{* *}$ & 0.509 & $0,625^{*}$ & $-0,017$ \\
& Signi. (p) & 0.002 & 0.010 & 0.091 & 0,022 & 0,957 \\
\hline Z-Beta & Pearson & -0.451 & $0.813^{* *}$ & $0.627^{*}$ & 0,495 & $-0,251$ \\
& Signi. (p) & 0.122 & 0.005 & 0.029 & 0,086 & 0,409 \\
\hline Z-Total & Pearson & 0.408 & $-0.647^{*}$ & $0.607 *$ & 0,473 & 0,010 \\
& Signi. (p) & 0.166 & 0.017 & 0.036 & 0,103 & 0,974 \\
\hline
\end{tabular}

The most significant values are indicated: * significant correlation at the 0.05 level; ** Significant at the 0.01 correlation.

\section{Discussion and conclusions}

One of the problems traditionally found in EEG studies on neuropsychiatric diseases of complex etiology is that, despite the electrophysiological patterns likely been significantly altered, it is very difficult to interpret the categories of change if they are not accompanied by other evidence and indicators that help make sense of the patient conditions. In view of this study on fibromyalgia patients, we can point to both psychological factors related to the dimension of perceived pain and catastrophization, and to physiological factors related to the physical condition of the patient, the severity of symptoms, and the influence of medication. Hence the importance of exploring in a systematic way the different types of evidence that can be obtained accompanying EEG recordings.

In that sense, the present pilot study is not obviously one of the first to consider quantitative EEG scans to examine brain wave patterns characteristic of fibromyalgia. But it has been done in a pretty thorough way. It has examined a sample of 26 subjects (13 patients and 13 controls), the scores of absolute powers and their corresponding Z-scores, the ratios of absolute powers theta vs. alpha and beta vs. alpha, the frequency peak of absolute power of the alpha band and of the total spectrum.

Some of the psychological outcomes were scarcely surprising. As pointed out in the scientific literature ${ }^{29,30}$, patients with fibromyalgia also experience depression, as happened with the participants of the study. However, regarding the catastrophizing variable (PCS), no statistically significant differences between patients and controls were found in this study. And this finding is unusual, since patients with chronic pain, as a group, tend to have higher levels of catastrophizing than healthy individuals, the fact that they are in drug treatment, mostly with antidepressants, modifies the basal levels of catastrophism and approaches them to normality. This is in agreement with the results obtained by ${ }^{31}$. Further, the sample size is relatively small so the dif- 
ferences between groups must be bigger than usual to be statistically significant.

For the different EEG indicators explored in the 13 patients, Pearson correlations have been obtained, both between indicators themselves and with the results of psychological tests. They emphasize the correlations between catastrophizing and Z-scores for absolute powers in the beta band $(0.627 ; \mathrm{p}=0.029)$ and total $(0.607 ; \mathrm{p}=0.036)$. The theta vs. alpha ratio correlated significantly with Z-delta PA $(-0.753 ; p=0.003)$, apart obviously from its correlation with Z- alpha and Z-theta PA. The beta vs. alpha ratio correlates with Zdelta $(-0.597 ; \mathrm{p}=0.031)$ and Z-theta $(-0.612$; $\mathrm{p}=0.026)$. The peak in the total spectrum correlates with Z-alpha $(0.625 ; \mathrm{p}=0.022)$. See Table 6 for the set of correlations. Not unexpectedly, the most relevant data collected is the statistical significance of the ratios between alpha and the two neighboring bands, theta and beta, in the comparison between patients and controls.

What does the power shift from alpha to theta and beta mean in information/cognitive terms? In principle, as Paul L. Nunez ${ }^{32}$ has argued, the alpha frequency represents the foundations of the overall information processing of the brain, as well as the basic ability to coordinate coherently the exchange patterns and information flows throughout the cortex (it should be considered, thus, as "the resonant frequency characteristic of the neocortex"). Therefore, a lower level of alpha activity, which in this case is really significant, becomes an indicator of diminished possibilities of sensorimotor integration in brain processing, and the need of an extra effort dedicated to the attenuation of chronic pain sensation.

In general, given the complexity of the disease and the diversity of conditions accompanying it (together with the relatively small number of subjects studied), we cannot expect a strict linear correlation between the results associated with the various indicators and psychometric tests. In this case, patients with the worst conditions produced ratios in the extremes and low frequency peaks, but other patients in better conditions had a more dispersed distribution of indicators. As for medications, we followed the criteria of not interfering with ongoing treatments ${ }^{33}$. It is possible, as indicated in the literature that the use of antidepressants and other relaxant drugs (and many other varieties) interfere with the results of EEG, but in summary they are not clearly separated from the random occurrence regarding patient populations without medications ${ }^{21}$.

Furthermore, Jeffrey B. Hargrove, in his study conducted on 85 patients with $\mathrm{FM}^{21}$, concluded that the various drugs used to treat this condition showed no significant differences between patients to whom each drug was administered and patients who were not administered the drug; and those who exhibited EEG abnormalities not expected by random occurrence occurred irrespective of the specific drugs taken. This suggests that the use of drugs does not influence the emergence of specific EEG patterns, as they found in that study. However, it would be advisable to carry out more studies in which patients and controls, with larger samples, are grouped to remove any reasonable doubt.

On the one side, the results we have obtained corroborate important qualitative and quantitative aspects described in the above literature, such as the general decline of absolute power, changes to the slowing of the alpha band (deviation of its power to theta and delta), together with the relative increase of the beta band, and the presence of significant defects in frontal and prefrontal areas (although not only in them). On the other side, unlike some related works ${ }^{19-21}$, herein we have defined ratios between bands theta 
and beta to alpha, not derived from Z-scores, but directly calculated from the values of absolute power; and they have been used to compare with controls and for the characterization of each individual patient, so to establish correlations with other neuropsychiatric and psychometric data. This aspect is an original feature of our study, and perhaps the most interesting result obtained. It amplifies the differences between patients and controls, by counting with two indicative ratios instead of only one-and, precisely, our use of the theta vs. alpha ratio, to our knowledge original in the EEG literature on FM, incorporates the most significant variations and correlations. Besides, by working directly with the absolute powers and not with Zscores, the calculation procedure is simplified, making it more real and more independent of any population reference.

We think these results are promising, and we are considering its continuation in two directions: for supplementing an ongoing study on coherence of phase (cordance) and evoked potentials, with records from the same population as the present work; and secondly, for a future increase of the sample size in order to achieve results of greater statistical power and to cross the indicators obtained from the EEG, not only with the psychological and neuropsychiatric tests, but also with the medication use already mentioned.

The main limitations of the study are: it is not a multicenter study, the sample size is small because this is an exploratory study, and the sample was recruited from a patient organization, not in primary care, so it might not be quite representative of people who suffer from this disease.

The EEG exploration continues, thus, showing its usefulness and its qualities of versatility, low cost, non-invasiveness, and ability to discriminate. It is a convenient tool for neurological exploration even in very complex diseases, such as fibromyalgia.

\section{Acknowledgements}

This study has been funded by project PI12/01480 (Instituto de Salud Carlos III) and by FEDER funds: "Una manera de hacer Europa". The Aragon Association of Fibromyalgia and Chronic Fatigue (ASAFA) helped to recruit the patients and controls for this study.

\section{References}

1. Clauw DJ. Fibromyalgia: a clinical review. Jama. 2014; 311(15): 1547-1555.

2. Valverde M, Juan A, Ribas B, Benito Urbina JC, Carmona I, and grupo de trabajo EPISER. Prevalencia de la fibromialgia en la población española. Estudio EPISER 2000. Rev Esp Reumatol. 2000; 27: 157.

3. Mas AJ, Carmona L, Valverde M, Ribas B. Prevalence and impact of fibromyalgia on function and quality of life in individuals from the general population: results from a nationwide study in Spain. Clin Exp Rheumatol. 2008; 26(4): 519-26.

4. Wolfe F, Clauw DJ, Fitzcharles MA, Goldenberg DL, Katz RS, Mease P, et al. The American College of Rheumatology preliminary diagnostic criteria for fibromyalgia and measurement of symptom severity. Arthritis Care Res. 2010; 62: 600-10.

5. Hardcastle VG. The Myth of Pain. Bradford Book. The MIT Press. Cambridge, MA. 1999.

6. Tyrer S. Psychosomatic pain. The British Journal of Psychiatry. 2006; 188(1): 91-93.

7. Greenberg DB, Braun IM, Cassem NH. Functional somatic symptoms and somatoform disorders. In: Stern TA, Rosenbaum JF, Fava M, Biederamn J, Rauch SL, eds. /Massachusetts General Hospital Comprehensive Clinical Psychiatry/. 1st ed. Philadelphia, Pa: Mosby Elsevier; 2008: chap 24.

8. Kringelbach ML, Jenkinson N, Owen SL, Aziz TZ. Translational principles of deep brain stimulation. Nat Rev Neurosci. 2007; 8(8): 623-35.

9. Legrain V, Iannetti GD, Plaghki L, Mouraux A. The pain matrix reloaded: a salience detection system for the body. Prog Neurobiol. 2011; 93(1): 111-24. 
10. Kuchinad A, Schweinhardt P, Seminowicz DA, Wood PB, Chizh BA, Bushnell MC. Accelerated brain gray matter loss in fibromyalgia patients: premature aging of the brain? J Neurosci. 2007; 27(15): 4004-7.

11. Garcia-Larrea L, \& Peyron R. Pain matrices and neuropathic pain matrices: a review. PAIN. 2013; 154: S29-S43.

12. Staud R. The Abnormal Central Pain Processing Mechanism In Patients With Fibromyalgia. Fibromyalgia Frontiers. 2002; 10(3): 18.

13. Loggia ML, Chonde DB, Akeju O, Arabasz G, Catana C, Edwards RR, et al. Evidence for brain glial activation in chronic pain patients. Brain. 2015; 138(3): 604-615.

14. DosSantos MF, Holanda-Afonso RC, Lima RL, DaSilva AF, Moura-Neto V. The role of the blood-brain barrier in the development and treatment of migraine and other pain disorders. Frontiers in cellular neuroscience. 2014; 8.

15. Crofford LJ, Demitrack MA. Evidence that abnormalities of central neurohormonal systems are key to understanding fibromyalgia and chronic fatigue syndrome. Rheum Dis Clin North Am. 1996; 22(2): 267-84.

16. Alp A, Oral AY, Ocako lu G, Dirican M, Yurtkuran M. Evidence of Defective Growth Hormone Response and Adaptive Hormonal Changes in Fibromyalgia. Physikalische Medizin, Rehabilitationsmedizin, Kurortmedizin. 2014; 24(01), 14-20.

17. Yunus MB. Dysfunctional Spectrum Syndrome: A Unified Concept For Many Common Maladies. Fibromyalgia Frontiers. 1996; 4(4): 3.

18. Woolf CJ. What to call the amplification of nociceptive signals in the central nervous system that contribute to widespread pain? Pain. 2014; 155(10): 1911-1912.

19. Donaldson M, Mueller H, Donaldson S, Sella G. QEEG patterns, psychological status and pain reports of fibromyalgia sufferers. Am. J. Of Pain Management. 2003; 13(2): 1-27.

20. Donaldson M \& Donalson S. The assessment of brain wave activity in fibromyalgia using quantitative electroencephalography techniques. Biofeedback. 2006; 34(3): 114-20.

21. Hargrove JB, Bennett RM, Simons DG, Smith SJ, Nagpal S, Deering DE. Quantitative electroencephalographic abnormalities in fibromyalgia patients. Clin EEG Neurosci. 2010; 41(3): 132-9.

22. Rosenfeld VW, Rutledge DN, Stern JM. Polysomnography with Quantitative EEG in Patients with and without Fibromyalgia. Journal of Clinical Neurophysiology. 2015; 32(2): 164-170.
23. Rodero B, García-Campayo J, Casanueva B, del Hoyo YL, Serrano-Blanco A, Luciano JV. Validation of the Spanish version of the Chronic Pain Acceptance Questionnaire (CPAQ) for the assessment of acceptance in fibromyalgia. Health Qual Life Outcomes. 2010; 8: 37.

24. García-Campayo J, Rodero B, Alda M, Sobradiel N, Montero J, Moreno S.Validation of the Spanish version of the Pain Catastrophizing Scale in fibromyalgia. Med Clin (Barc). 2008; 131(13): 487-92.

25. Spielberger C, Gorsuch R, Lushene R, Seisdedos Cubero N. STAI. Madrid: TEA; 2011.

26. Conde V, Useros E. Adaptación castellana de la escala de evaluación conductual para la depresión de Beck. Rev Psiquiatr Psicol Med Eur Am. 1975; 12: 217-236.

27. Alonso J, Prieto L, Antó JM. La versión española del SF-36 Health Survey (Cuestionario de Salud SF-36): Un instrumento para la medida de los resultados clínicos. Med Clin (Barc). 1995; 104: 771-76.

28. John ER, Prichep LS, Fridman J, Easton P. Neurometrics: computer-assisted differential diagnosis of brain dysfunctions. Science. 1988; 239: 162-9.

29. Okifuji A, Turk D, Sherman J. Fibromyalgia Syndrome. Why aren't all patients depressed? J Rheumatol. 2000; 27: 212-219.

30. Sansone RA, Levengood JV, Sellbom M. Psychological aspects of fibromyalgia: research vs. clinical impressions. J Psychosom Res. 2004; 56: 185-88.

31. Alda M, Luciano JV, Andrés E, Serrano-Blanco A, Rodero B, Del Hoyo YL, Roca M, Moreno S, Magallón R, García-Campayo J. Effectiveness of cognitive behaviour therapy for the treatment of catastrophisation in patients with fibromyalgia: a randomised controlled trial. Arthritis Res. Ther. 2011; 13(5): R173.

32. Nunez PL. Brain, Mind, and the Structure of Reality. New York: Oxford University Press. 2010.

33. Billiot K, Budzynski T \& Andrasik F. EEG patterns and chronic fatigue. Journal of Neurotherapy. 1997; 2(2): 20-30.

\footnotetext{
* Corresponding author:

Jorge Navarro López

Avda. San Juan Bosco 13 pl. 0

50009 Zaragoza

Spain

Tel. 0034976713526

Fax 0034976715554

E-mail: jnavarro.iacs@aragon.es
} 